\title{
Montenegro's skin reactions and antibodies against different Leishmania species in dogs from a visceral leishmaniosis endemic area
}

\author{
Carolina O. Baleeiro ${ }^{a}$, Moacir Paranhos-Silva ${ }^{\mathrm{b}}$, Juliana C. dos Santos ${ }^{\mathrm{a}}$, \\ Geraldo G.S. Oliveira ${ }^{\mathrm{a}}$, Eliane Goes Nascimento ${ }^{\mathrm{c}}$, Lain Pontes de Carvalho ${ }^{\mathrm{a}, \mathrm{d}}$, \\ Washington L.C. dos-Santos ${ }^{\mathrm{a}, \mathrm{d}, *}$ \\ ${ }^{a}$ Centro de Pesquisas Gonçalo Moniz-Fiocruz, Fundação Oswaldo Cruz, Rua Waldemar Falcão 121, 40295-001 Salvador (BA), Brazil \\ ${ }^{\mathrm{b}}$ Laboratório Central do Estado da Bahia (LACEN), Salvador, Brazil \\ ${ }^{\mathrm{c}}$ Centro de Referencia em Endemias Piraja da Silva (PIEJ), Jequie, Brazil \\ ${ }^{\mathrm{d}}$ Escola Bahiana de Medicina e Saúde Pública, Av. Dom João VI no. 274, Brotas, 40290-000 Salvador (BA), Brazil
}

Received 5 January 2005; received in revised form 21 February 2006; accepted 24 February 2006

\begin{abstract}
In this study, humoral (circulating anti-Leishmania antibodies) and cellular (Montenegro's skin test) immune responses of dogs from an endemic area of visceral leishmaniosis were tested using Leishmania chagasi, Leishmania amazonensis and Leishmania braziliensis antigens. The antibody response was tested in three animal groups, selected according to their anti- $L$. chagasi antibody activity, as measured by ELISA in the serum: 19 negative (O.D. below 0.30), seven with undefined (O.D. between 0.40 and 0.70 ) and 12 positive (O.D. above 1.0) ELISA result. In the group of animals with positive ELISA, the antibody activity against $L$. chagasi antigens (mean O.D. $=1.31$ ) was significantly higher (ANOVA, $P<0.01)$ than against $L$. amazonensis (mean O.D. $=0.88$ ) or L. braziliensis (mean O.D. $=0.87$ ) antigens. The Montenegro's skin test results obtained with $L$. chagasi and L. braziliensis antigens showed a fair agreement $(\kappa=0.309)$. The same was observed when antigens from $L$. braziliensis and L. amazonensis were compared $(\kappa=0.374)$, whereas a moderate agreement between the results from tests performed with $L$. chagasi and $L$. amazonensis antigens was observed $(\kappa=0.530)$. The induration areas obtained with $L$. braziliensis antigen were smaller than those obtained with the other antigens. The data presented herein indicate that the use of antigens from different Leishmania species may interfere with the results of the immunological tests performed in dogs in an endemic area of visceral leishmaniosis.
\end{abstract}

(C) 2006 Elsevier B.V. All rights reserved.

Keywords: Leishmanin skin test; Leishmania chagasi; Leishmania braziliensis; Leishmania amazonensis; Leishmaniasis; Antibody-response

* Corresponding author at: Centro de Pesquisas Gonçcalo Moniz - Fundação Oswaldo Cruz, Rua Waldemar Falcão 121, Candeal, 40296-710, Salvador (BA), Brazil. Tel.: +55 713176 2262; fax +55 7131762324 .

E-mail address: wluis@cpqgm.fiocruz.br (Washington L.C. dos-Santos). 


\section{Introduction}

The zoonotic visceral leishmaniosis is caused by Leishmania chagasi/Leishmania infantum (Grimaldi and Tesh, 1993). Dogs are believed to be the main reservoir of these parasites (Dye, 1996). The identification of infected dogs is not always an easy task: the clinical signs of the disease are non-specific and the current gold standard diagnosis for visceral leishmaniosis is the identification of parasites in tissues (Barrouin-Melo et al., 2004). The accomplishment of this, however, is invasive, time consuming and influenced by factors such as parasite burden, the stage of disease development and the specific requirements of some parasite strains for growing in culture (Zijlstra et al., 1998; Braz et al., 2002; Desjeux, 2004). Hence, a variety of simple immunological tests are used to characterize infection and disease. In general, most of these tests have a good performance for the diagnosis of disease (Cabrera et al., 2003), but they are less sensitive for the detection of infection (Paranhos-Silva et al., 2003; Ashford et al., 1995). Some authors have reported an increase in the sensitivity of anti-Leishmania immunological tests by using the parasite strains (Badaro et al., 1983; Badaro et al., 1986) or species (Reed et al., 1986) associated with the infection as source of antigens.

In the diagnostic routine, however, Leishmania species differing from those implicated in the genesis of disease are used as source of antigens (Paranhos-Silva et al., 2001; Zijlstra et al., 2001; EL-Safi et al., 2002; Gavgani et al., 2002; Borges et al., 2003). The reasons for such practice are (1) the wide cross-reactivity of the immune responses against different Leishmania species and (2) the costs inherent to antigen production and quality control of the antigen batches.

In the present work, we examined the effect of using antigens of different Leishmania species in tests for humoral and cellular immune responses in dogs from an endemic area of visceral leishmaniosis. The question dealt with in this work is if the results obtained in these tests are comparable.

\section{Materials and methods}

\subsection{Animals}

A total of 154 mixed-bred stray dogs, non-infected or naturally infected with Leishmania, were captured in the streets of the urban area of Jequie municipality, State of Bahia, Brazil, at different periods, from 1997 to 2001. Jequie is an endemic area of visceral and tegumentar leishmanioses (Dos Santos et al., 1993; Paranhos-Silva et al., 1996). Visceral leishmaniosis is found in the urban area of the municipality (Dos Santos et al., 1993) and L. chagasi was the only species isolated from dogs (Paranhos-Silva et al., 1996) and human beings (Paranhos-Silva et al., 2003) with the disease. Cutaneous and mucocutaneous leishmaniosis is found in the countryside, about $20 \mathrm{~km}$ from the urban center, and Leishmania braziliensis has been isolated from human beings with the disease (de Oliveira et al., 2003). The dogs were identified with sequential numbers and were only included in the study if they were not claimed by putative owners during a legal 2-day period. Clinical signs of disease were recorded, a sample of blood was collected for serological tests, Montenegro's skin test (MST) was performed and the animals were sacrificed with lethal injection of potassium chloride. During the sacrifice spleen aspirates were collected for culture. Not all animals were subjected to all the tests.

\subsection{Clinical evidence of disease}

Presence or absence of clinical signs of disease such as emanciation, skin lesions such as eczema or ulcers and corneal opacification was recorded for 145 out of the 154 animals.

\subsection{Parasite antigens}

Leishmania amazonensis (MHOM/BR/88/BA125), Leila strain, was gently provided by Dr. Aldina Barral, Centro de Pesquisas Gonçalo Moniz, Fundação Oswaldo Cruz, Salvador, Brazil; L. braziliensis (MOM3456, isolated from a human case of cutaneous leshmaniosis) was a gift from Dr. Margarida Pompeu, Federal University of Ceará, Brazil and L. chagasi (MHOM/BR2000/Merivaldo2) was isolated from a patient with visceral leishmaniasis, from Jequie, Brazil (Paranhos-Silva et al., 2001). All the parasites were characterized by isoenzymatic profile and reactivity with monoclonal antibody by Dr. Gabriel Grimaldi, Oswaldo Cruz Institute, FIOCRUZ, Rio de Janeiro. Stationary-phase promastigotes of the parasites were grown in a Schneider's medium containing 
$20 \%(\mathrm{v} / \mathrm{v})($ L. braziliensis) or $10 \%(\mathrm{v} / \mathrm{v})$ (the other species) foetal bovine serum (FBS). They were washed three times with $0.15 \mathrm{M}$ phosphate-buffered saline, $\mathrm{pH}$ 7.4 (PBS) and lysed by repeated cycles of freezing and thawing followed by sonication. Antigen batches to be used in ELISA were processed in the presence of a combination of enzymatic inhibitors: $2 \mu \mathrm{g} / \mathrm{ml}$ phenylmethanesulfonyl fluoride (PMSF), $2 \mu \mathrm{g} / \mathrm{ml} N$ - $p$-tosylL-phenylalanine chloromethyl ketone (TPCK), $2 \mu \mathrm{g} / \mathrm{ml}$ $N$ alfa- $p$-tosyl-L-lisyne chloromethyl ketone (TLCK), $2 \mu \mathrm{g} / \mathrm{ml}$ 4-nitrophenyl $p$-guanidinobenzoate (NPGB) and $2 \mu \mathrm{g} / \mathrm{ml} \quad N$-(trans-epoxysuccinyl)-L-leucine 4 guanidinobutylamide; trans-3-carboxyoxirane-2-carbonyl-L-leucylagmatine; trans-epoxysuccinyl-L-leucylamido (4-gunidino) butane; L-trans-3-carboxyoxiran2-carbonyl-L-leucylagmatine (E64). No enzyme inhibitors or preserving agents were added to the antigen batches used for the Montenegro's skin test. Antigen suspensions were stored at $-70{ }^{\circ} \mathrm{C}$ until use.

\subsection{Comparison of antigens from different \\ Leishmania species for the detection of antibodies in serum}

For this part of the study we reviewed the results of an ELISA, which employed L. chagasi antigen (Paranhos-Silva et al., 2003; see details below), previously used for the diagnosis of canine leishmaniasis as part of a surveillance program in stray dogs in the Municipality of Jequie. Sera from 38 dogs with different intensities of anti-L. chagasi antibody activity in the ELISA were selected in order to set up groups of sera representative of three non-overlapping categories: (i) 19 sera producing O.D. equal or below 0.3 (i.e. producing negative ELISA results), (ii) seven sera producing O.D. between 0.4 and 0.7 (producing what was considered an undefined ELISA result), and (iii) 12 animals producing O.D. equal or higher than 1.0 (i.e. leading to a positive ELISA result, with high antibody activity). Dogs with ELISA O.D. values equal or above 1.0 were frequently found infected with Leishmania and animals with ELISA O.D. below 0.4 were almost always found non-infected with Leishmania, when examined by spleen culture. In order to allow a more accurate comparison among groups, each serum was tested in duplicate and the results confirmed in two independent repetitions. Assays for the different antigens were run in the same microtiter plate (32 wells of each plate were sensitized with each Leishmania lysate). Serum samples of animals from each group were run in parallel, in the same plate, in each assay. Briefly, flat-bottom 96-well microtiter plates (Cliniplate-Labsystem) were coated with $100 \mu \mathrm{l}$ of L. amazonensis $(15 \mu \mathrm{g} / \mathrm{ml})$, L. braziliensis $(15 \mu \mathrm{g} /$ $\mathrm{ml})$ or L. chagasi $(15 \mu \mathrm{g} / \mathrm{ml})$ antigen in carbonate buffer (0.06 M, pH 9.6). The optimal concentration of each antigen for plate sensitization was determined in preliminary assays using serum from animals experimentally infected with the different Leishmania species. Non-specific reactions were blocked with a solution of PBS containing 10\% (w/v) powdered skimmed milk. After washing with PBS containing $0.05 \%$ (v/v) Tween 20 (PBS-Tween), serum samples, diluted 1:400 and 1:1000 in ELISA buffer [PBS containing $10 \%(\mathrm{w} / \mathrm{v})$ of powdered skimmed milk and $0.05 \%$ (v/v) Tween-20] were added to each well. Horseradish peroxidase-sheep anti-dog IgG conjugates (Bethyl Laboratories) were used at a dilution of 1:1000 (Paranhos-Silva et al., 2003). The reactions were developed as described elsewhere (dos Santos et al., 1996).

\subsection{Montenegro's skin test}

This was performed in 143 out of the 154 animals: 39 were tested with $L$. amazonensis, $L$. braziliensis and $L$. chagasi antigens and the other 104 animals were tested with L. braziliensis and L. chagasi antigens only. All antigens were diluted in saline to a final concentration of $2.5 \mathrm{mg}$ of protein/ml. Each animal was intradermally injected with $100 \mu \mathrm{l}$ volumes of each antigen at different sites of the abdominal skin (Paranhos-Silva et al., 2001). As a control, $100 \mu \mathrm{l}$ of the diluent (saline) was injected at a different site of the abdominal skin. Forty-eight hours after the injections, the sizes of indurations were measured by observers without previous knowledge of the site of injections. The results are expressed as the mean of the largest diameter of the induration area. Skin reactions with diameter equal or larger than $5 \mathrm{~mm}$ were considered positive.

\subsection{Parasite isolation in cultures}

Immediately after the sacrifice, the spleens of the animals were aseptically exposed and samples were collected by puncture. Spleen aspirates were cultured 
in biphasic (agar-blood-Schneider medium). Cultures were examined weekly for at least 2 months for the presence of parasites. This procedure was performed in 143 out of the 154 animals.

\subsection{Expression and analysis of the results}

The data shown in the tables represent absolute numbers, proportions or mean \pm standard deviations of measurements obtained from animals of each group. The statistical significance of the differences observed among groups was tested using one-way analysis of variance (ANOVA). When the $F$-test was found significant, the difference between groups was identified using the Student-Newman-Keuls' test. For cross-tables comparisons, Fisher's exact probability test was used. Concordance between categorical results of the tests was evaluated using the kappa coefficient (calculated according to Landis and Koch) (Landis and Koch, 1977). The $\kappa$ statistic, a measure of the agreement between two observers or tests, ranges from -1 to 1 with 1 indicating perfect agreement, 0 indicating the agreement expected on the basis of chance alone. It has been proposed the following as standards for strength of agreement for the kappa coefficient: $0=$ poor, $0.01-0.20=$ slight, $0.21-0.39$ = fair, $0.40-0.60=$ moderate, $0.61-0.80=$ substantial, and $0.81-1=$ almost perfect (Seigel et al., 1992). The critical level of significance admitted was 0.05 (Glantz, 1997).

\section{Results}

\subsection{Anti-L. chagasi, -L. braziliensis and -L. amazonensis antibody activities in serum}

Differences in serum antibody activities against $L$. chagasi, L. braziliensis and $L$. amazonensis antigens were statistically significant only in the group of animals with positive anti-L. chagasi ELISA (Table 1). In these animals, antibody activities in 1:400-diluted sera were higher (ANOVA, followed by SNK test, $P<0.01)$ against $L$. chagasi $(1.442 \pm 0.184)$ than against $L$. braziliensis $(1.210 \pm 0.346)$ or against $L$. amazonensis (1.154 \pm 0.327$)$ antigens. At the dilution

Table 1

Anti-Leishmania antibody activity in the serum and response to Montenegro's skin test (MST) measured in dogs from an endemic area for visceral leishmaniosis using antigens of different Leishmania species

\begin{tabular}{|c|c|c|c|}
\hline \multirow[t]{2}{*}{ Parameters } & \multicolumn{3}{|c|}{ Source of antigen } \\
\hline & L. chagasiis & L. braziliensis & L. amazonens \\
\hline \multicolumn{4}{|l|}{ Antibody activity in serum at $1: 400$ dilution } \\
\hline Negative $^{\mathrm{a}}(n=19)$ & $0.228 \pm 0.053$ & $0.228 \pm 0.057$ & $0.204 \pm 0.082$ \\
\hline Undefined $^{\mathrm{b}}(n=7)$ & $0.526 \pm 0.113$ & $0.445 \pm 0.145$ & $0.426 \pm 0.146$ \\
\hline $\operatorname{Positive}^{\mathrm{c}}(n=12)$ & $1.442 \pm 0.184^{\mathrm{d}}$ & $1.210 \pm 0.346$ & $1.154 \pm 0.327$ \\
\hline \multicolumn{4}{|l|}{ Antibody activity in serum at $1: 1000$ dilution } \\
\hline Negative $(n=19)$ & $0.133 \pm 0.027$ & $0.148 \pm 0.068$ & $0.113 \pm 0.026$ \\
\hline Undefined $(n=7)$ & $0.271 \pm 0.078$ & $0.225 \pm 0.065$ & $0.228 \pm 0.069$ \\
\hline Positive $(n=12)$ & $1.308 \pm 0.200^{\mathrm{e}}$ & $0.868 \pm 0.334$ & $0.878 \pm 0.330$ \\
\hline \multicolumn{4}{|l|}{ Montenegro's skin test } \\
\hline Proportion of animals with positive $\mathrm{MST}^{\mathrm{f}}$ & $22 / 143(15 \%)^{\mathrm{g}}$ & $10 / 143(7 \%)$ & $4 / 39(10 \%)^{\mathrm{h}}$ \\
\hline Induration area $(\mathrm{mm})$ & $9.3 \pm 2.0$ & $7.5 \pm 2.7$ & $8.3 \pm 2.2$ \\
\hline
\end{tabular}

Mean optical density \pm standard deviation of the mean.

${ }^{\text {a }}$ Dogs with negative results in the ELISA (O.D. $\leq 0.3$ ) using $L$. chagasi antigen.

${ }^{\mathrm{b}}$ Dogs with undefined results in the ELISA (O.D. =0.4-0.7) using L. chagasi antigen.

${ }^{c}$ Dogs with positive results in the ELISA (O.D. $\left.\geq 1.0\right)$ using L. chagasi antigen.

${ }^{\mathrm{d}}$ Significantly different from the other antigens (ANOVA, followed by SNK test, $P<0.01 ; P<0.001$, respectively).

e Significantly different from the other antigens (ANOVA, followed by SNK test, $P<0.01 ; P<0.001$, respectively).

f Animals with induration area $\geq 5 \mathrm{~mm}$.

${ }^{\mathrm{g}}$ Six out of these 22 animals also had MST positive with L. braziliensis antigen.

${ }^{\mathrm{h}}$ One of the animals also had positive MST with $L$. chagasi and another with both $L$. chagasi and L. braziliensis antigens. 
of 1:1000, serum antibody activity was higher (ANOVA, followed by SNK test, $P<0.001$ ) against L. chagasi $(1.308 \pm 0.200)$ than against $L$. braziliensis $(0.868 \pm 0.334)$ or against $L$. amazonensis $(0.878 \pm$ 0.330 ) antigens. There were no statistically significant differences between serum anti-Leishmania antibody activities against antigens of $L$. braziliensis and $L$. amazonensis. In the group of animals with undefined (O.D. $=0.4-0.7)$ anti-L. chagasi ELISA the antibody activity in the serum was slightly higher to $L$. chagasi than to the other Leishmania antigens. Such difference was not, however, statistically significant. Furthermore, the O.Ds. were substantially lower in four animals when tested with $L$. amazonensis than when tested with L. chagasi antigen: in two animals the O.D. changed from $>1.0$ with $L$. chagasi to $0.7-0.4$ with $L$. amazonensis, and in two others the O.D. changed from 0.7 to 0.4 with $L$. chagasi to $<0.3$ with $L$. amazonensis. Three of these animals also had O.D. readings lower with $L$. braziliensis than with $L$. chagasi.

\subsection{Montenegro's skin test}

Thirty-five out of 143 (24\%) animals developed induration areas of $2.5-12.5 \mathrm{~mm}$ at the sites of injection of $L$. chagasi antigen. The test was, however, considered positive (with indurations areas equal or larger than $5 \mathrm{~mm})$ in $22(15 \%)$ of these animals (Table 1). The average diameter of the induration areas of the positive reactions was $8.6 \pm 2.3 \mathrm{~mm}$. Nineteen out of $143(13 \%)$ animals developed induration areas of 3-13 $\mathrm{mm}$ at the sites of injection of L. braziliensis antigen. The test was positive in $10(7 \%)$ of these animals. The average diameter of the induration areas of the positive reactions was $7.5 \pm 2.8 \mathrm{~mm}$. Four out of $39(10 \%)$ animals developed induration areas of $6.5-12 \mathrm{~mm}$ at the sites of injection of $L$. amazonensis antigen. The average diameter of the induration areas of the positive reactions was $9.2 \pm 2.3 \mathrm{~mm}$ (Table 1).

Among the 143 animals tested with $L$. chagasi and L. braziliensis antigens, $16(11 \%)$ were positive for $L$. chagasi; 4 (3\%) were positive for L. braziliensis; 6 (4\%) was positive for both $L$. chagasi and $L$. braziliensis and $117(82 \%)$ were negative for both antigens. The kappa coefficient of agreement between tests using L. chagasi or L. braziliensis antigens was 0.309 . Among the 39 of these 143 animals which had also been tested with $L$. amazonensis antigen, one had positive MST with the three antigens, one had positive MST with L. chagasi and L. amazonensis, one had positive MST with $L$. chagasi only, two had positive MST with L. amazonensis only and 34 had negative MST. The kappa coefficient of agreement between tests using $L$. chagasi or L. amazonensis antigens was 0.530 and using $L$. braziliensis or L. amazonensis antigens was 0.374 .

\subsection{Clinical signs of disease}

Clinical signs of disease were present in 69 animals and absent in 76. In the animals which were selected as blood donors for the comparative analysis of different Leishmania antigens in ELISA, clinical signs of disease were present in all of the 12 animals in the category with positive ELISA, in three out of the seven animals in the category with undefined ELISA and in eight out of 15 animals (from which clinical data were recorded) in the category with negative ELISA. The difference in the frequencies of animals with clinical signs of disease between the group with high antibody activity and the other groups was statistically significant $(P=0.006$, Fisher's exact probability test).

Clinical signs of disease were recorded from 136 out of the 143 animals subjected to MST with $L$. chagasi and L. braziliensis antigens, including the 39 animals also tested with $L$. amazonensis antigen. Clinical signs of disease were present in 64 of these animals. Nine out of 64 animals (14.1\%) with clinical signs of disease had positive MST. Among the 72 animals without clinical signs of disease, 18 had positive MST (25.0\%). This difference in the presence of clinical signs of disease observed in animals with and without positive MST was not statistically significant.

\subsection{Presence of parasites in the spleen}

Cultures were positive in 29 animals and negative in 114 animals. Leishmania was detected in 11 out of 11 tested animals in the category with positive serum anti- $L$. chagasi antibody activity and in two out of seven in the category with undefined anti-L. chagasi ELISA result. None of the 15 animals tested in the category with negative serum anti-L. chagasi antibody activity had parasites in their spleen cultures. The difference in the frequencies of positive spleen 
cultures between the group with positive ELISA and the other groups was statistically significant ( $P=0.0001$, Fisher's exact probability test).

Splenic cultures for detection of Leishmania were performed in 134 out of the 143 animals subjected to MST with $L$. chagasi and L. braziliensis antigens, including the 39 animals also tested with $L$. amazonensis antigen. The cultures were positive in 25 of these 134 animals: five with positive MST to L. chagasi antigen only and 20 with negative MST.

\section{Discussion}

This work formally demonstrates that variations in the Leishmania species used for antigen preparation may significantly influence the results of the tests for the diagnosis and evaluation of the immune response of dogs from an endemic area of visceral leishmaniosis. Using antigen preparations made from $L$. amazonensis or L. braziliensis instead of L. chagasi (the species associated with infection in the endemic area under study) resulted in a decrease in the intensity of antibody activity measured in the serum of the animals in an ELISA. It is well known that, when antiparasite antibody serological tests are performed in animals from an endemic area of leishmaniosis or other parasitic diseases, one usually finds three groups of animals in terms of how their serological results can be interpreted: (i) one with well defined negative results, (ii) one with well defined positive results, and (iii) a third, usually small group of animals with undefined test results (which might be said to be in the "gray zone"). The working hypothesis of the present work was that it is easier to identify minor quantitative differences in the antigenicity of different Leishmania species when sera from this third group of animals, rather than sera producing clearly negative or clearly positive results, were used in the serological test. Although in this work statistically significant differences were only found in the group of animals with high anti- $L$. chagasi antibody levels, the assay that used $L$. amazonensis antigen produced O.D. readings below 0.3 in two animals of the group with undefined results. That assay would therefore define those sera, perhaps erroneously, as clearly negative. Similar observations concerning better results obtained with homologous than with heterologous antigens were made by da Costa and collaborators in animals experimentally infected with $L$. chagasi and tested against antigens of $L$. chagasi and Leishmania mexicana (da Costa et al., 1991). Also Badaro et al. (1986), in a study performed on human beings, found that lysates from strains of $L$. chagasi coming from the same region of the serum donors, when used as antigen, produced stronger reactions in an ELISA than lysates of $L$. chagasi from other region. The same group, however, found little differences in the sensitivities of immunofluorescence (Badaro et al., 1983) and of ELISA (Badaro et al., 1986) performed with sera from patients suspected of being infected by $L$. chagasi, when promastigotes of $L$. chagasi or amastigotes of L. mexicana were used as antigen in the tests. The data presented herein suggest that L. amazonensis and L. braziliensis promastigotes would have a lower performance in serological surveys carried out in endemic areas of visceral leishmaniosis. Further studies are needed, however, to assess the actual differences in the sensitivity and specificity of tests performed using antigens from Leishmania species non-implicated as causative agent of canine visceral leishmaniosis.

Discrepancies were also observed in the results of the MST. The strength of the agreement between the tests using $L$. chagasi or L. braziliensis was fair and between tests using $L$. chagasi or $L$. amazonensis antigens was moderate. In spite of the small number of animals tested with the three antigens, the differences in performance were more evident between $L$. chagasi and $L$. braziliensis than between $L$. chagasi and L. amazonensis. It is important, however, to note that the intensity of the LST reactions were high with L. amazonensis antigen even in the two animals that were negative to $L$. chagasi antigens. One of the reasons that may explain this observation is that, as mentioned above, Jequie is an endemic region for visceral and cutaneous leishmaniosis (Dos Santos et al., 1993; Paranhos-Silva et al., 1996). Visceral leishmaniosis has a urban distribution, while cutaneous leishmaniosis occurs in some rural areas (Dos Santos et al., 1993). Although the dogs evaluated in this study, with positive test against Leishmania were probably infected by L. chagasi, we cannot exclude the possibility of some of the animals being infected by $L$. amazonensis or L. braziliensis during putative trips to the countryside. Unfortunately the characteristics of the study did not 
allow recording data on the origin of these animals or on trips outside the urban area of Jequie.

Most of the studies on comparisons of antigens used in MST performed on human beings revealed antigen-dependent differences in outcomes. For example, Reed et al. (1986) found that a lysate of $L$. chagasi led to higher sensitivity than lysates of $L$. amazonensis in MST performed in individuals with past history of visceral leishmaniosis. Ben Alaya Bouafif and collaborators (Ben Alaya Bouafif et al., 2001) found only a moderate concordance level between MST performed using L. infantum and Leishmania major antigens in an endemic area for visceral leishmaniosis. The authors however, attributed the antigen-associated discrepancies observed in the results to possible technical problems arising during antigen injection. Jorquera and collaborators (Jorquera et al., 1998), examining human beings from an endemic area for visceral and tegumentary leishmaniosis, also found individuals that selectively responded to preparations containing $L$. chagasi (8.7\%) or to preparations containing a mixture of $L$. amazonensis, Leishmania venezuelensis and L. braziliensis $(23.9 \%)$, or to preparations containing both antigens $(67.4 \%)$. Such variety of responses found in human beings is similar to that observed in this study with dogs. Similar observations were made by Agwale and collaborators (Agwale et al., 1998), working with different populations of the same endemic area of cutaneous leishmaniasis suggest that the sensitivity of MST may be increased when a pool of heterologous antigens instead of a single heterologous antigen Leishmania is used in the preparation.

In the study reported herein most animals (15\%) had a positive MST to L. chagasi antigen. However, six out of 121 animals (5\%) that had a negative skin reaction to $L$. chagasi had a positive reaction to $L$. amazonensis or L. braziliensis antigens. The overall positivity, when we consider a positive response against at least one of the three antigens, would therefore be higher than the positivity to any of the antigens. To identify all dogs with a delayed-type hypersensitivity skin reaction to Leishmania antigens in Jequie, therefore, it may be desirable to test antigens from different Leishmania species, separately or mixed in just one preparation.

Another data that emerged from the present work, confirming data formerly presented in the literature, is the association of active parasitism in the spleen and presence of clinical signs of disease in animals with high antibody activity in the serum (Paranhos-Silva et al., 2003). Also, a smaller proportion of animals with positive MST had spleen parasitism and clinical signs of disease than the animals with negative MST. Although such difference did not reach statistical significance, it may be indicative of MST as a potential marker of cell-mediated immunity in L. chagasiinfected dogs, as proposed by other authors (Pinelli et al., 1994).

In conclusion, using antigens from different species of Leishmania for diagnosis and assessment of the immune response in animals from an endemic area of visceral leishmaniosis may lead to non-comparable results. The differences in the performance of the tests using different parasite species observed in this study, together with observations reported in the literature, may be taken into account in the designing of new tests for diagnosis or for assessing the canine immune response in leishmaniosis. This is particularly true if these tests are designed for general use or in regions where more than one parasite species is present. The characteristics of the cross-reactive immune response observed between Leishmania species in this and other studies may also be taken into consideration in the design of new vaccines against canine visceral leishmaniosis.

\section{Acknowledgments}

This work was supported by the Brazilian Research Council (CNPq, grant no. 473048/03-5) and Bahia State Research Support Foundation (FAPESB, grant no. 114/2002). We are in debit with Dr. Edson Duarte Moreira for his careful review of the statistical analysis of the data presented herein.

\section{References}

Agwale, S.M., Duhlinska, D.D., Grimaldi Jr., G., 1998. Response to heterologous leishmanins in cutaneous leishmaniasis in Nigeria-discovery of a new focus. Mem. Inst. Oswaldo Cruz. 93, 23-27.

Ashford, D.A., Bozza, M., Freire, M., Miranda, J.C., Sherlock, I., Eulalio, C., Lopes, U., Fernandes, O., Degrave, W., Barker Jr., R.H., et al., 1995. Comparison of the polymerase chain reaction 
and serology for the detection of canine visceral leishmaniasis. Am. J. Trop. Med. Hyg. 53, 251-255.

Badaro, R., Reed, S.G., Barral, A., Orge, G., Jones, T.C., 1986. Evaluation of the micro enzyme-linked immunosorbent assay (ELISA) for antibodies in American visceral leishmaniasis: antigen selection for detection of infection-specific responses. Am. J. Trop. Med. Hyg. 35, 72-78.

Badaro, R., Reed, S.G., Carvalho, E.M., 1983. Immunofluorescent antibody test in American visceral leishmaniasis: sensitivity and specificity of different morphological forms of two Leishmania species. Am. J. Trop. Med. Hyg. 32, 480-484.

Barrouin-Melo, S.M., Larangeira, D.F., Trigo, J., Aguiar, P.H., dosSantos, W.L., Pontes-de-Carvalho, L., 2004. Comparison between splenic and lymph node aspirations as sampling methods for the parasitological detection of Leishmania chagasi infection in dogs. Mem. Inst. Oswaldo Cruz. 99, 195-197.

Ben Alaya Bouafif, N., Ben Salah, A., Louzir, H., Zaatour, A., Bel Haj Hamida, N., Mighri, F., Gharbi, A., Gramiccia, M., Gradoni, L., Dellagi, K., Ben Ismail, R., 2001. Concordance of leishmaniasis cutaneous tests in Tunisia. Arch. Inst. Pasteur Tunis. 78, $11-16$.

Borges, V.C., Ruiz, M.C., Gomes, P.M., Colombo, A.R., Silva Lde, A., Romero, H.D., Prata, A., 2003. Montenegro intradermoreaction after the test sequential repetitions in Porteirinha, Minas Gerais State, Brazil. Rev. Soc. Bras. Med. Trop. 36, 249-251.

Braz, R.F., Nascimento, E.T., Martins, D.R., Wilson, M.E., Pearson, R.D., Reed, S.G., Jeronimo, S.M., 2002. The sensitivity and specificity of Leishmania chagasi recombinant K39 antigen in the diagnosis of American visceral leishmaniasis and in differentiating active from subclinical infection. Am. J. Trop. Med. Hyg. 67, 344-348.

Cabrera, M.A., Paula, A.A., Camacho, L.A., Marzochi, M.C., Xavier, S.C., da Silva, A.V., Jansen, A.M., 2003. Canine visceral leishmaniasis in Barra de Guaratiba, Rio de Janeiro, Brazil: assessment of risk factors. Rev. Inst. Med. Trop. Sao Paulo 45, 79-83.

da Costa, C.A., Genaro, O., de Lana, M., Magalhaes, P.A., Dias, M., Michalick, M.S., Melo, M.N., da Costa, R.T., Magalhaes-Rocha, N.M., Mayrink, W., 1991. Canine visceral leishmaniasis: evaluation of the serologic method used in epidemiologic studies. Rev. Soc. Bras. Med. Trop. 24, 21-25.

de Oliveira, C.I., Bafica, A., Oliveira, F., Favali, C.B., Correa, T., Freitas, L.A., Nascimento, E., Costa, J.M., Barral, A., 2003. Clinical utility of polymerase chain reaction-based detection of Leishmania in the diagnosis of American cutaneous leishmaniasis. Clin. Infect. Dis. 37, e149-e153.

Desjeux, P., 2004. Leishmaniasis: current situation and new perspectives. Comp. Immunol. Microbiol. Infect. Dis. 27, 305-318.

Dos Santos, A.J., Nascimento, E.G., Silva, M.P., De Carvalho, L.C., 1993. Report on a visceral and cutaneous leishmaniases focus in the town of Jequie, State of Bahia, Brazil. Rev. Inst. Med. Trop. Sao Paulo 35, 583-584.

dos Santos, W.L., Rahman, J., Klein, N., Male, D.K., 1996. Control of lymphocyte adhesion to brain and aortic endothelium: ICAM1, VCAM-1 and negative charge. J. Neuroimmunol. 66, 125134.
Dye, C., 1996. The logic of visceral leishmaniasis control. Am. J. Trop. Med. Hyg. 55, 125-130.

EL-Safi, S.H., Bucheton, B., Kheir, M.M., Musa, H.A., EL-Obaid, M., Hammad, A., Dessein, A., 2002. Epidemiology of visceral leishmaniasis in Atbara River area, eastern Sudan: the outbreak of Barbar El Fugara village (1996-1997). Microbes Infect. 4, 1439-1447.

Gavgani, A.S., Hodjati, M.H., Mohite, H., Davies, C.R., 2002. Effect of insecticide-impregnated dog collars on incidence of zoonotic visceral leishmaniasis in Iranian children: a matchedcluster randomised trial. Lancet 360, 374-379.

Glantz, S.A., 1997. Primer of Bio-Statistics, 4th ed. McGraw-Hill, New York/San Francisco, pp. 1-473.

Grimaldi Jr., G., Tesh, R.B., 1993. Leishmaniases of the New World: current concepts and implications for future research. Clin. Microbiol. Rev. 6, 230-250.

Jorquera, A., Ledezma, E., De Sousa, L., Garcia, A., Sanchez, J., Zerpa, J., Gonzalez, R., O’Daly, J.A., 1998. Epidemiologic characterization of American cutaneous leishmaniasis in an endemic region of eastern Venezuela. Am. J. Trop. Med. Hyg. 58, 589-593.

Landis, J.R., Koch, G.G., 1977. The measurement of observer agreement for categorical data. Biometrics 33, 159-174.

Paranhos-Silva, M., Freitas, L.A., Santos, W.C., Grimaldi, G.J., Pontes-de-Carvalho, L.C., Oliveira-dos-Santos, A.J., 1996. A cross-sectional serodiagnostic survey of canine leishmaniasis due to Leishmania chagasi. Am. J. Trop. Med. Hyg. 55, 39-44.

Paranhos-Silva, M., Oliveira, G.G., Reis, E.A., de Menezes, R.M., Fernandes, O., Sherlock, I., Gomes, R.B., Pontes-de-Carvalho, L.C., dos-Santos, W.L., 2003. A follow-up of Beagle dogs intradermally infected with Leishmania chagasi in the presence or absence of sand fly saliva. Vet. Parasitol. 114, 97-111.

Paranhos-Silva, M., Pontes-de-Carvalho, L.C., de Sa Oliveira, G.G., Nascimento, E.G., dos-Santos, W.L., 2001. Skin reactions to thimerosal and Leishmania in dogs from a leishmaniasis endemic area: it is better to keep them apart. Mem. Inst. Oswaldo Cruz. 96, 679-681.

Pinelli, E., Killick-Kendrick, R., Wagenaar, J., Bernadina, W., del Real, G., Ruitenberg, J., 1994. Cellular and humoral immune responses in dogs experimentally and naturally infected with Leishmania infantum. Infect. Immun. 62, 229-235.

Reed, S.G., Badaro, R., Masur, H., Carvalho, E.M., Lorenco, R., Lisboa, A., Teixeira, R., Johnson Jr., W.D., Jones, T.C., 1986. Selection of a skin test antigen for American visceral leishmaniasis. Am. J. Trop. Med. Hyg. 35, 79-85.

Seigel, D.G., Podgor, M.J., Remaley, N.A., 1992. Acceptable values of kappa for comparison of two groups. Am. J. Epidemiol. 135, 571-578.

Zijlstra, E.E., Daifalla, N.S., Kager, P.A., Khalil, E.A., El-Hassan, A.M., Reed, S.G., Ghalib, H.W., 1998. rK39 enzyme-linked immunosorbent assay for diagnosis of Leishmania donovani infection. Clin. Diagn. Lab. Immunol. 5, 717-720.

Zijlstra, E.E., Nur, Y., Desjeux, P., Khalil, E.A., El-Hassan, A.M., Groen, J., 2001. Diagnosing visceral leishmaniasis with the recombinant K39 strip test: experience from the Sudan. Trop. Med. Int. Health 6, 108-113. 